\title{
Dietary carbohydrates: a review of international recommendations and the methods used to derive them
}

\author{
A. E. Buyken ${ }^{1,2}$ - D. J. Mela ${ }^{3}$ P. Dussort ${ }^{4}$ • I. T. Johnson ${ }^{5}$ - I. A. Macdonald ${ }^{6}$ J. D. Stowell ${ }^{7}$ F. J. P. H. Brouns ${ }^{8}$
}

Received: 6 April 2017 / Revised: 18 September 2017 / Accepted: 5 October 2017 / Published online: 25 April 2018

(c) The Author(s) 2018. This article is published with open access

\begin{abstract}
Background/objectives Renewed dietary recommendations for carbohydrates have recently been published by various international health authorities. The present work (1) reviews the methods and processes (systematic approach/review, inclusion of public consultation) used to identify, select and grade the evidence underpinning the recommendations, particularly for total carbohydrate (CHO), fibre and sugar consumption, and (2) examines the extent to which variation in the methods and processes applied relates to any differences in the final recommendations.

Subjects/methods A search of WHO, US, Canada, Australia and European sources identified 19 documents from 13 authorities with the desired detailed information. Processes and methods applied to derive recommendations were compiled and compared.

Results (1) A relatively high total CHO and fibre intake and limited intake of (added or free) sugars are generally recommended. (2) Even where recommendations are similar, the specific justifications for quantitative/qualitative recommendations differ across authorities. (3) Differences in recommendations mainly arise from differences in the underlying definitions of $\mathrm{CHO}$ exposure and classifications, the degree to which specific CHO-providing foods and food components were considered, and the choice and number of health outcomes selected. (4) Differences in the selection of source material, time frames or data aggregation and grading methods appeared to have minor influence.

Conclusions Despite general consistency, apparent differences among the recommendations of different authorities would likely be minimized by: (1) More explicit quantitative justifications for numerical recommendations and communication of uncertainty, and (2) greater international harmonization, particularly in the underlying definitions of exposures and range of relevant nutrition-related outcomes.
\end{abstract}

Electronic supplementary material The online version of this article (https://doi.org/10.1038/s41430-017-0035-4) contains supplementary material, which is available to authorized users.

\footnotetext{
P. Dussort

info@ilsieurope.be

1 Institute of Nutrition, Consumption and Health, Faculty of Natural Sciences, University Paderborn, Paderborn, Germany

2 DONALD Study Dortmund, Department of Nutritional Epidemiology, University of Bonn, Bonn, Germany

3 Unilever R\&D Vlaardingen, Vlaardingen, The Netherlands

4 International Life Sciences Institute-ILSI Europe a.i.s.b.l., Avenue E. Mounier 83, Box 6, Brussels, Belgium
}

\section{Introduction}

In addition to comprehensive evidence-based guidance on nutrient intakes for the prevention of nutrition-related diseases from the World Health Organization (WHO) [1-3], many national and regional bodies also publish quantitative nutrient recommendations (NR) and/or qualitative or quantitative food-based guidelines (FBG). These form a

5 Quadram Institute Bioscience, Norwich Research Park, Norfolk NR4 7UA, UK

6 School of Life Sciences, Queen's Medical Centre, University of Nottingham, Nottingham, UK

7 DuPont Nutrition \& Health, Danisco Ltd, Reigate, UK

8 Faculty of Health, Medicine and Life Sciences, Department of Human Biology, Maastricht University, Maastricht, The Netherlands 
basis for public food and health, policies or nutrition education programmes and to help citizens make healthy food and beverage choices. In addition, they are often the basis for implementation of governmental actions, including food labelling and public health campaigns, particularly with regard to consumption of carbohydrates ( $\mathrm{CHO}$ ), including added or free sugars. Some differences in NR or FBG may be justified by consideration of the specific target population. However, if variations in guidance reflect arbitrary differences in process and methodology, this may create or amplify an unjustified impression of inconsistencies in the scientific evidence itself

In order to evaluate the nature and sources of variation in NRs and FBG, the first aim of the present work was to review current quantitative NRs for $\mathrm{CHO}$, focused particularly on dietary fibre, sugars and total $\mathrm{CHO}$, to identify consistencies and inconsistencies across these. In addition, we have also reviewed recommendations related to glycaemic index and load (GI, GL) and whole grain. As some authorities also published FBGs or documents comprising both NRs and FBGs, FBGs were also considered if they referred to major CHO-providing foods (e.g. Australian [4] or Dutch [5] documents). Most of the NRs/FBGs draw on comprehensive reports appraising the underlying evidence base. Hence, the second aim was to review the methodological approaches used to select and extract the evidence base for the respective reports and to examine how differences in the resultant NRs/FBGs may relate to variation in aspects of the methodological approaches. In contrast to a recent review by Erickson et al. [6] on sugars guidelines, the objective was not to assess the quality of the methodology or evidence base underpinning current recommendations but to review the overall approach followed by different authorities, in order to consider the extent to which this may have resulted in variation in the resulting recommendations.

\section{Methods}

\section{Selection and identification of NRs/FBGs and associated reports}

Relevant NRs/FBGs and their related reports published in English from January 2000 through January 2016 were sought for the following organizations, regions, and countries: WHO, United States of America (US), Canada, Australia, and countries of the European Union (EU) and European Free Trade Association (the 28 EU countries plus Iceland, Liechtenstein, Norway, and Switzerland), Russia and Turkey. To address aim 1 (review of current NRs/ FBGs), inclusion criteria were availability of NRs/FBGs in English from an authoritative national/regional body for intake of dietary fibre, sugars, or total $\mathrm{CHO}$, as well as whole grain, glycaemic index (GI) or glycaemic load (GL). To address aim 2 (review of NRs/FBGs methodology), we further identified whether an evidence-based report including a description of the specific methodology used to derive the NRs/FBGs was available from the respective country/ region. Justifications for recommendations focused on dietary fibre and sugar, but also total $\mathrm{CHO}, \mathrm{GI} / \mathrm{GL}$ and whole grains were extracted from the NRs/FBGs themselves or the underlying reports. In some cases secondary sources of information were included, such as articles published in other languages (Dutch and German). Papers were excluded if they did not provide either NRs/FBGs or a report on the evidence base. Where evidence was duplicated (e.g., in reviews), the latest and original documents were used.

From personal knowledge and contact with international nutrition authorities, the research team was already aware of relevant documents from Australia, Germany, The Netherlands, Nordic region (combined), United Kingdom (UK), The US, Canada, the European Food Safety Authority (EFSA, for the EU) and the WHO [2, 4, 5, 7-22]. To supplement this, a systematic approach was undertaken to identify additional relevant documents published in English from the remaining countries, by applying the search strategy outlined in Appendix 1 using the OvidSP platform and MEDLINE, FSTA, and CAB abstracts databases. This search returned 1781 titles and abstracts, which were independently reviewed by pairs of co-authors of the current paper. From these, 27 papers were identified by at least one co-author as potentially relevant for inclusion as primary source material. In a further step, these full papers were accessed and independently reviewed by three co-authors. Of these full texts, three papers (one for Ireland, two for Spain) provided NRs/FBGs data [23-25]. The other 24 documents identified from the search were rejected because they were general commentaries, proposed (but not officially adopted) guidance, dietary intake assessments or evaluations, other survey data, guidelines for the management of a disease or at-risk population or not available in English.

\section{Extraction of data from NRs/FBGs and reports}

For the overview of $\mathrm{CHO}$ NRs/FBGs from different authorities, data were extracted from the corresponding papers. Recommended intakes of total $\mathrm{CHO}$, sugars and fibre were extracted in detail (Tables 1, 2 and 3). For a more in-depth analysis of the NRs/FBGs on intakes of dietary sugar(s) and fibre, the justifications given by the different authorities were also extracted. Finally, NRs/FBGs on whole grain, GI, and GL were extracted for a narrative summary. 


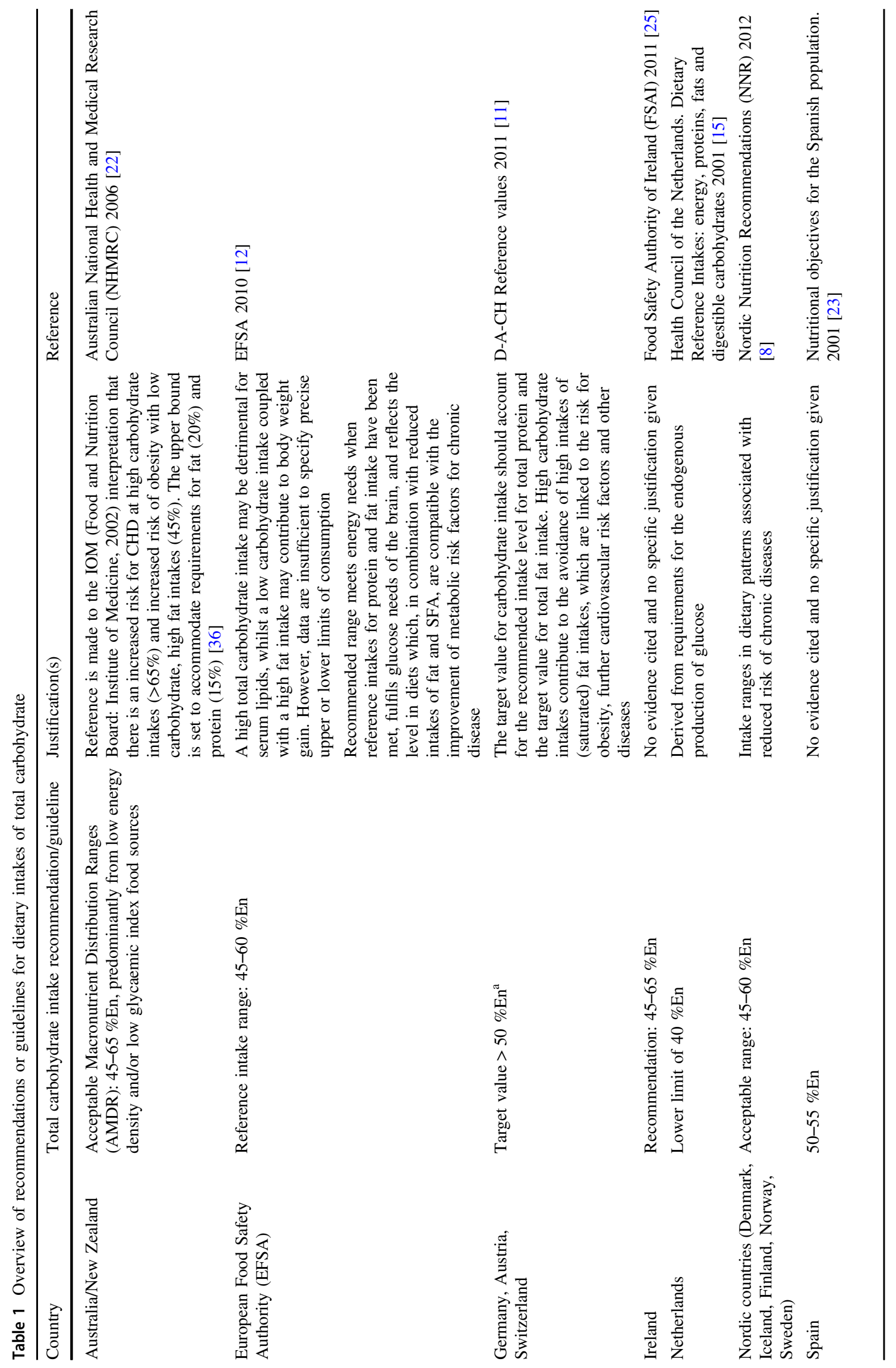




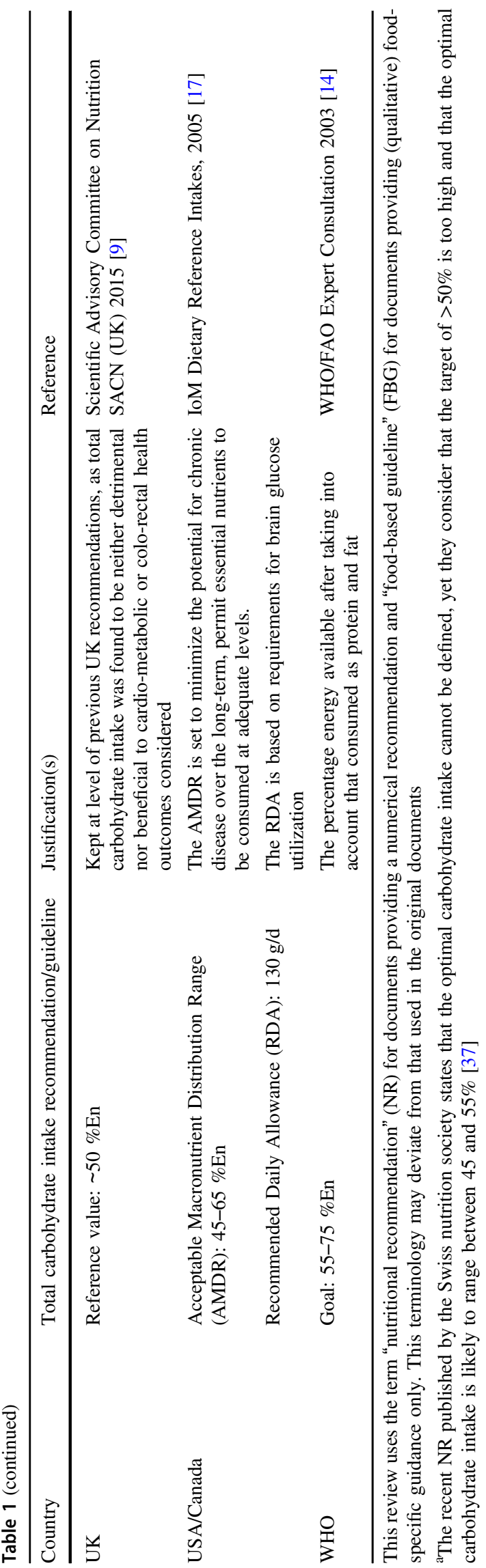

For the overview of methodologies employed for the reports (Table 4), detailed information was extracted from papers describing the evidence-based approaches applied (see Appendix 2).

Regardless of the original language and terms, we refer to "NRs" where the scientific evidence has been translated into conclusions expressed in explicit amounts (grams, percent energy, etc.) of carbohydrate components, and "FBGs" where this is expressed in portions or more qualitative terms for intakes of certain types or groups of carbohydrate-containing foods and beverages.

\section{Results}

\section{NRs/FBGs and justifications}

\section{Total carbohydrate}

Overall, $11 \mathrm{NRs/FBGs} \mathrm{on} \mathrm{total} \mathrm{CHO}$ intake from ten authorities were identified, comprised of one global (WHO), five regional (EFSA, D-A-CH, Nordic countries, USA/ Canada, Australia/New Zealand) and four national recommendations (Table 1). All authorities recommend a total $\mathrm{CHO}$ intake approaching or exceeding half the percentage total energy intake (\%En); however, the exact numbers/ ranges differ amongst authorities, ranging broadly from a "lower limit" of $40 \% \mathrm{En}$ [15] to 55-75 \% En [14]. There is correspondingly wide variation in the justifications given for these values, in part reflecting differences between minimal physiological requirements and the (higher) levels deemed optimal for disease risk reduction.

Related to this, the terminology used to describe the recommended range or value also differs. While some simply state a recommended value/range, others use specific terms with different definitions including "reference intake/ value" [9, 12], "target value" [11], "goal" [14], "population range" [8], or "acceptable macronutrient distribution" [17, 22]. The rationale for and meaning of these terms are generally explained in the documents, and are used in part to differentiate between the expression of nutritional requirements and levels advised for optimal health (disease risk reduction) in the population.

\section{Sugars}

Several reports provide only qualitative statements regarding limitation of sugar consumption and/or of foods providing (added) sugars [4, 5, 11, 23-25] (Table 2). The 2010 EFSA report [12] concluded that there are insufficient data to set an upper limit for (added) sugar intake. Similarly, the Institute of Medicine concluded in 2005 [17] that there were insufficient data for an upper level for added sugars and 


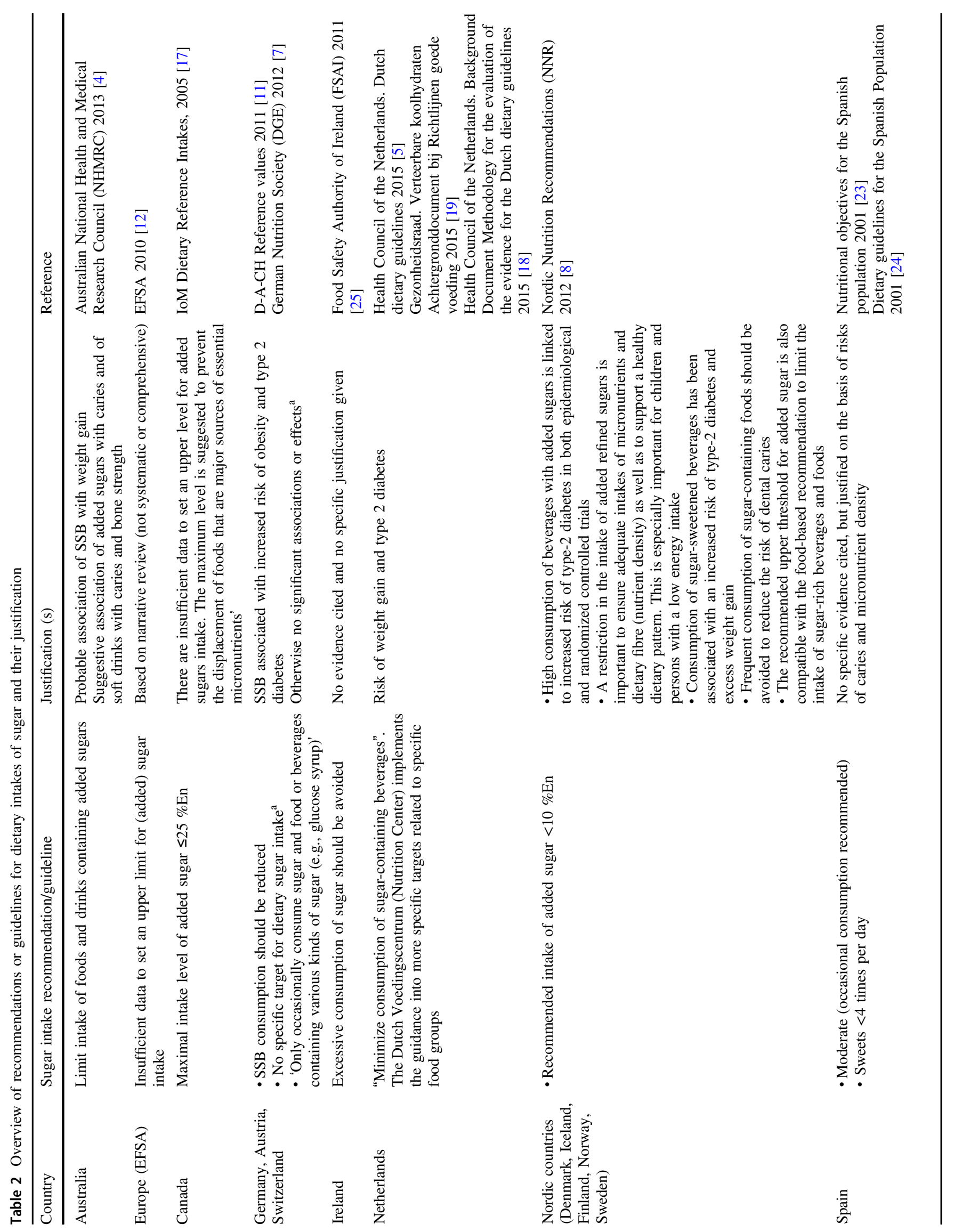




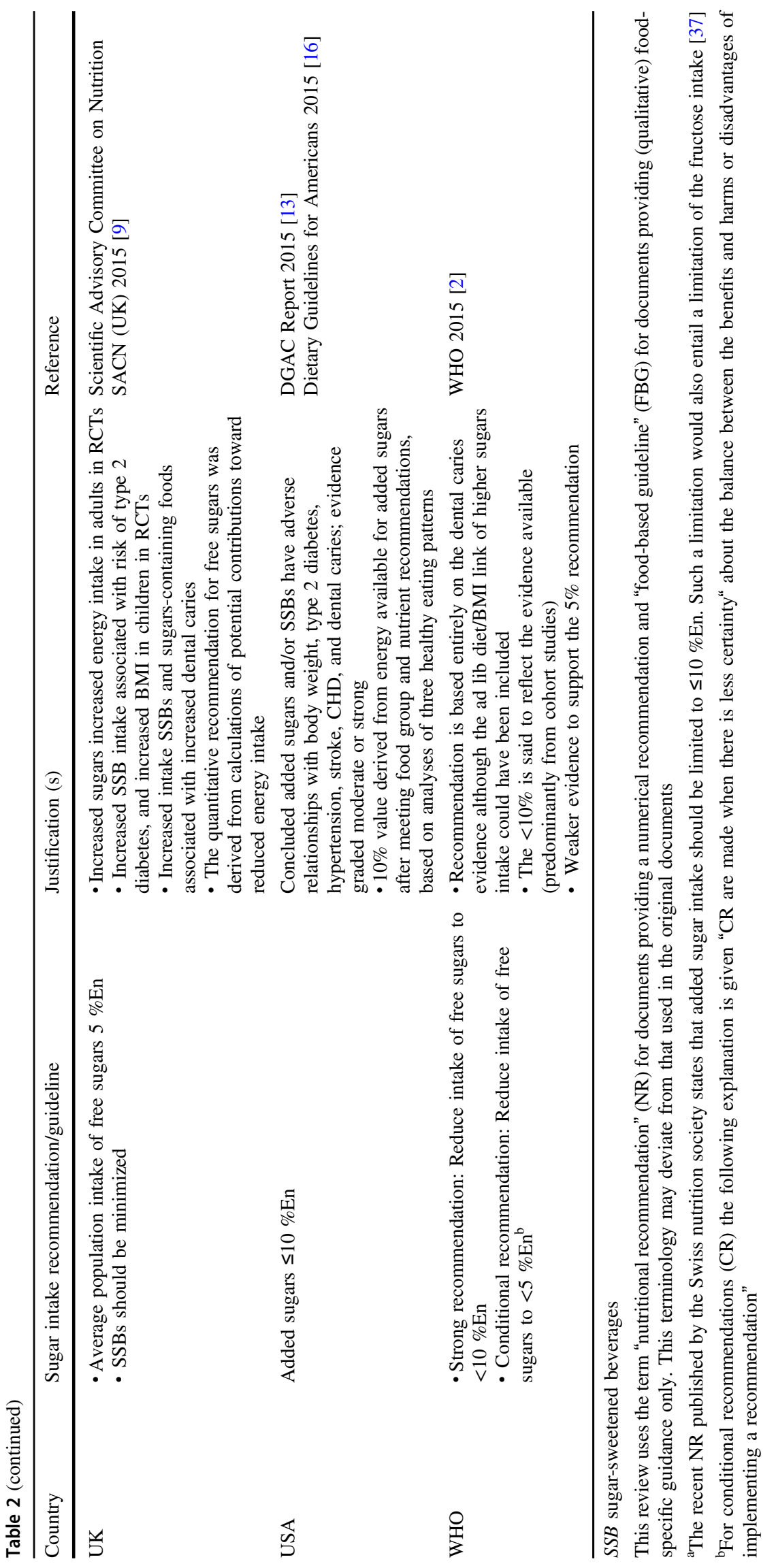




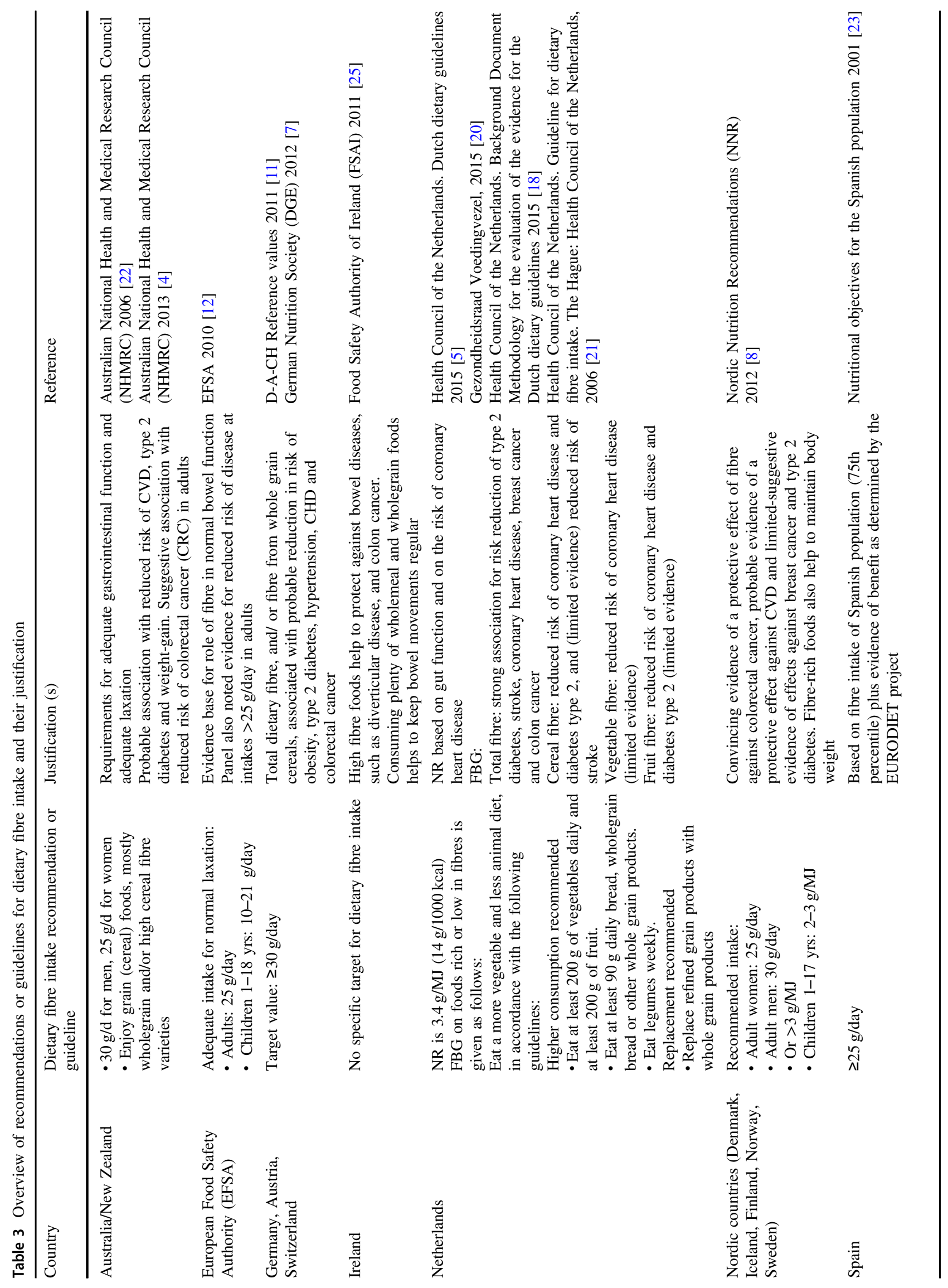




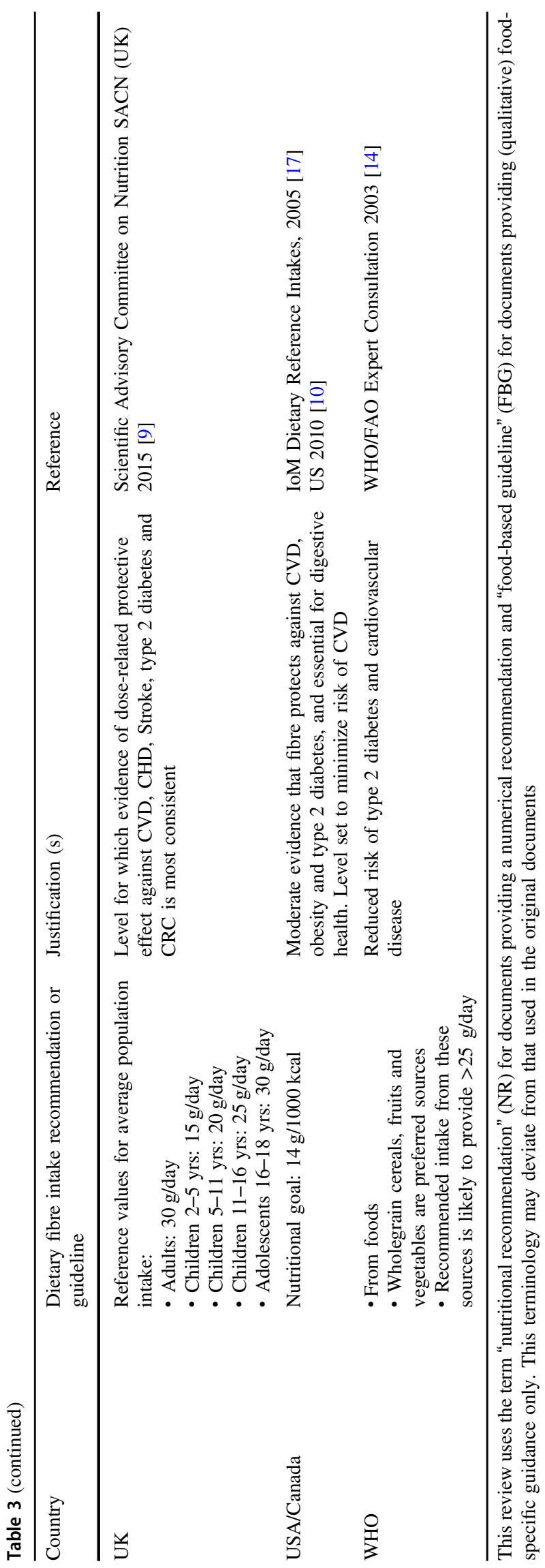

suggested a maximal intake level of $\leq 25 \%$ En "to prevent the displacement of foods that are major sources of essential micronutrients". In contrast, several more recent NRs specifically advise intakes of added or free sugars $\leq 10 \%$ En [2, $8,13]$. The WHO additionally gives a conditional NR to reduce free sugar consumption to levels $<5 \% \mathrm{En}$ [2]. A level of $<5 \%$ En from free sugars was also recommended for the UK by The Scientific Advisory Committee on Nutrition (SACN) [9], based on consideration of elevated risks of dental caries and excessive energy intake and weight gain with free sugars in general, and type 2 diabetes with intakes of sugar sweetened beverages (SSBs).

As with total CHO, the terminology, and thus the sources of sugars highlighted in the NRs/FBGs differ among the different authorities. Advice may be food-specific, or directly related to "sugar", "added sugar" or "free sugar" intakes. In this respect it should be noted that "added" and "free" are not the same thing, but differ mainly by inclusion of naturally occurring sugars in fruit juices under the latter term (Table 5).

There is pronounced variation in the justification for the quantitative sugar intake recommendations, even where the recommendations themselves are similar (Table 2). In some cases there is very little indication of how the NRs or FBGs were derived [23-25].

Other authorities give explicit justifications based primarily on evidence for increased disease risk associated with higher intakes of SSBs [4, 7]. The reports of the UK (SACN) [9], the USA (DGAC) [13] and the WHO [2] relate a number of disease risks to higher added or free sugars from foods and beverages, but the outcomes explicitly used as the basis for quantitative recommendations differ for each authority. WHO [2] described relationships of free sugar intakes with both body weight and dental caries, but based their quantitative NRs only on caries. The UK [9] recommendations note relationships of free sugars with risks of dental caries, type 2 diabetes (for SSBs) and increased energy intake, with the latter being the primary basis for deriving a quantitative recommendation. The 2015 US DGAC report [13] refers to a number of associations of sugar intakes with risk of various non-communicable diseases, but the quantitative recommendation for added sugars was based on implications for meeting preferred dietary patterns. The Nordic recommendations also justify advice for reduced added sugar intakes with reference to dental caries, SSBs in relation to diabetes and the importance of ensuring adequate micronutrient and fibre intake [8].

\section{Dietary fibre}

Recommendations for fibre intakes are in a relatively narrow range across the various authorities, with intake levels for adults generally given as $\geq 25$ or $\geq 30$ g/day or the 


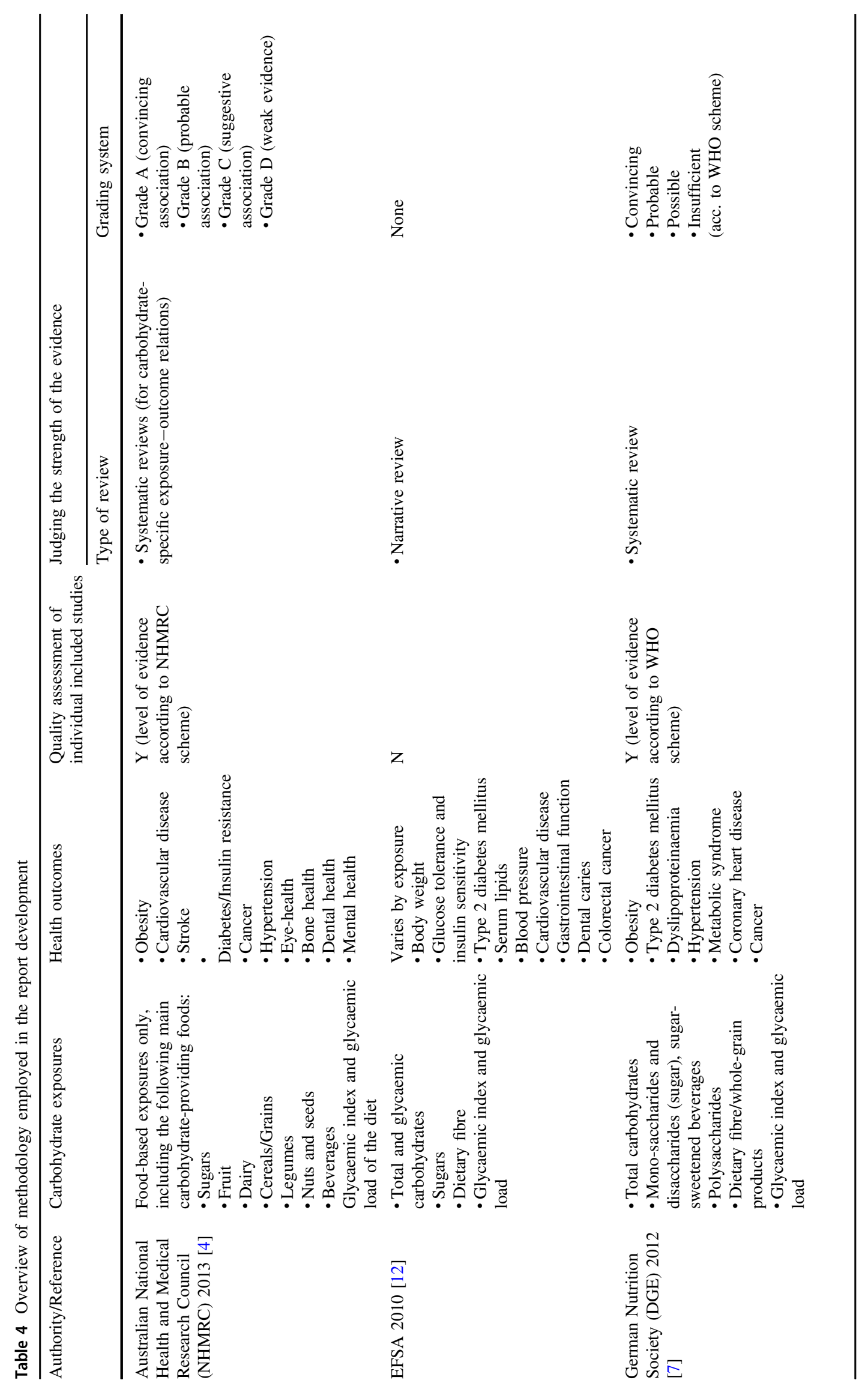




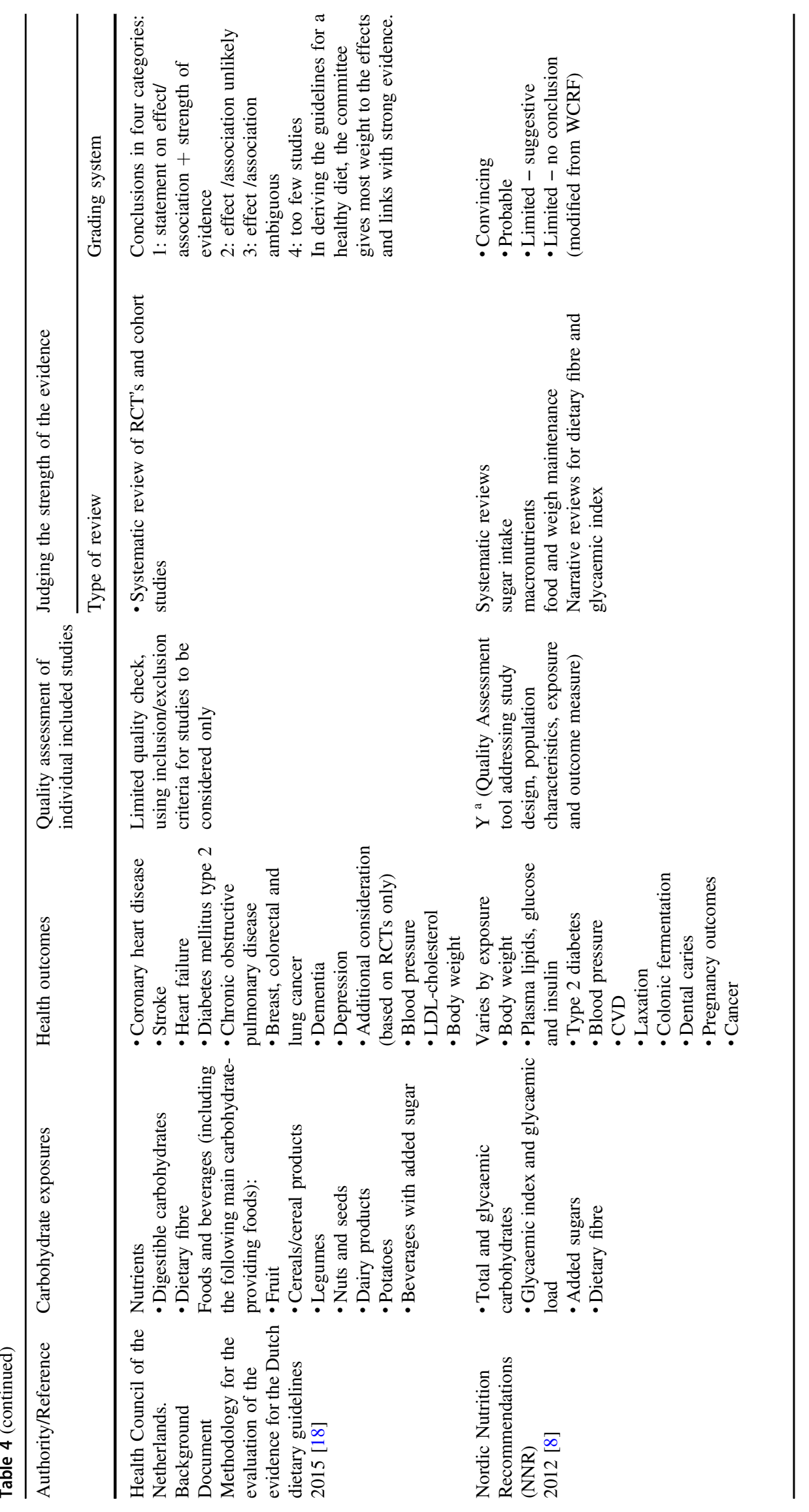




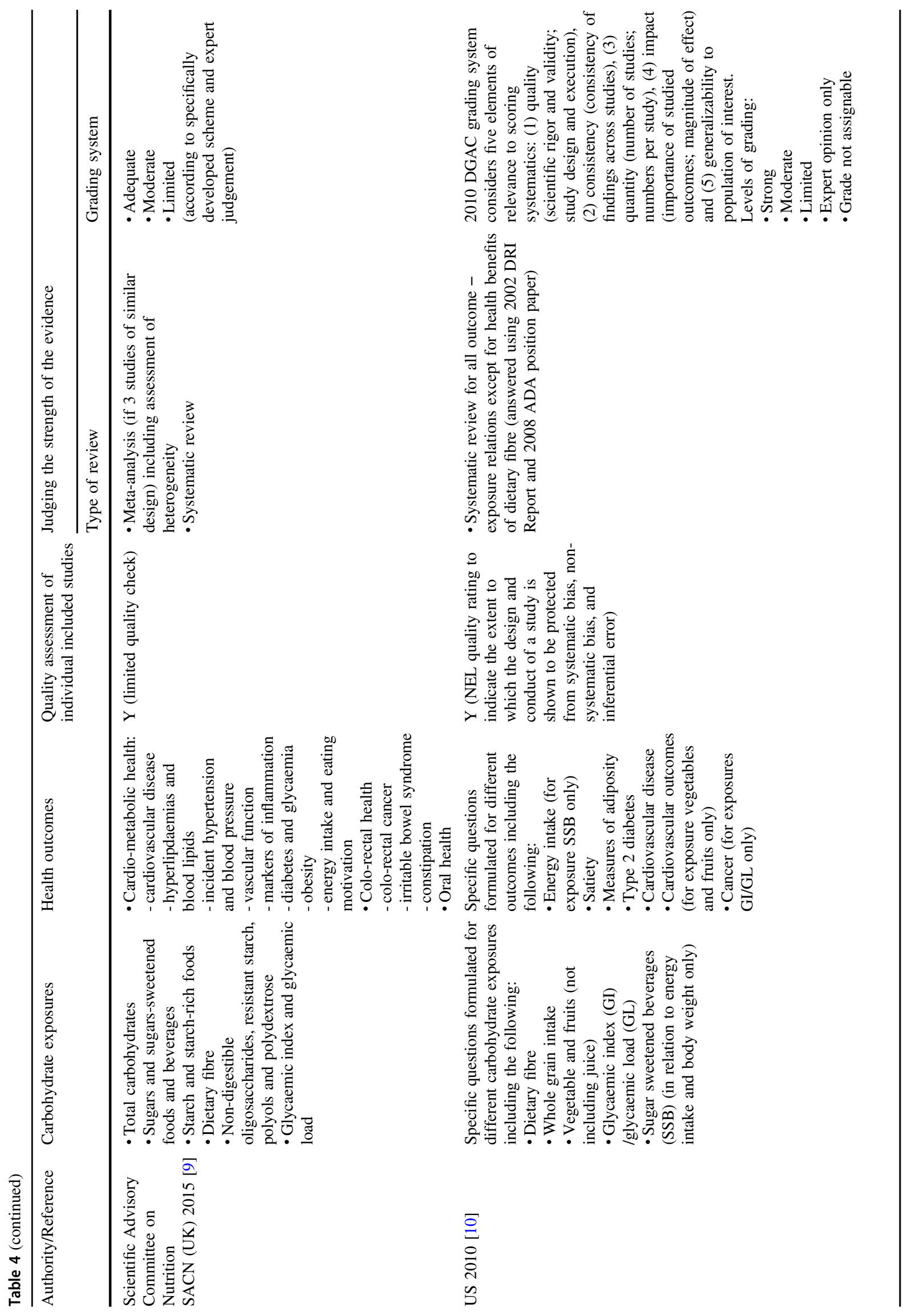




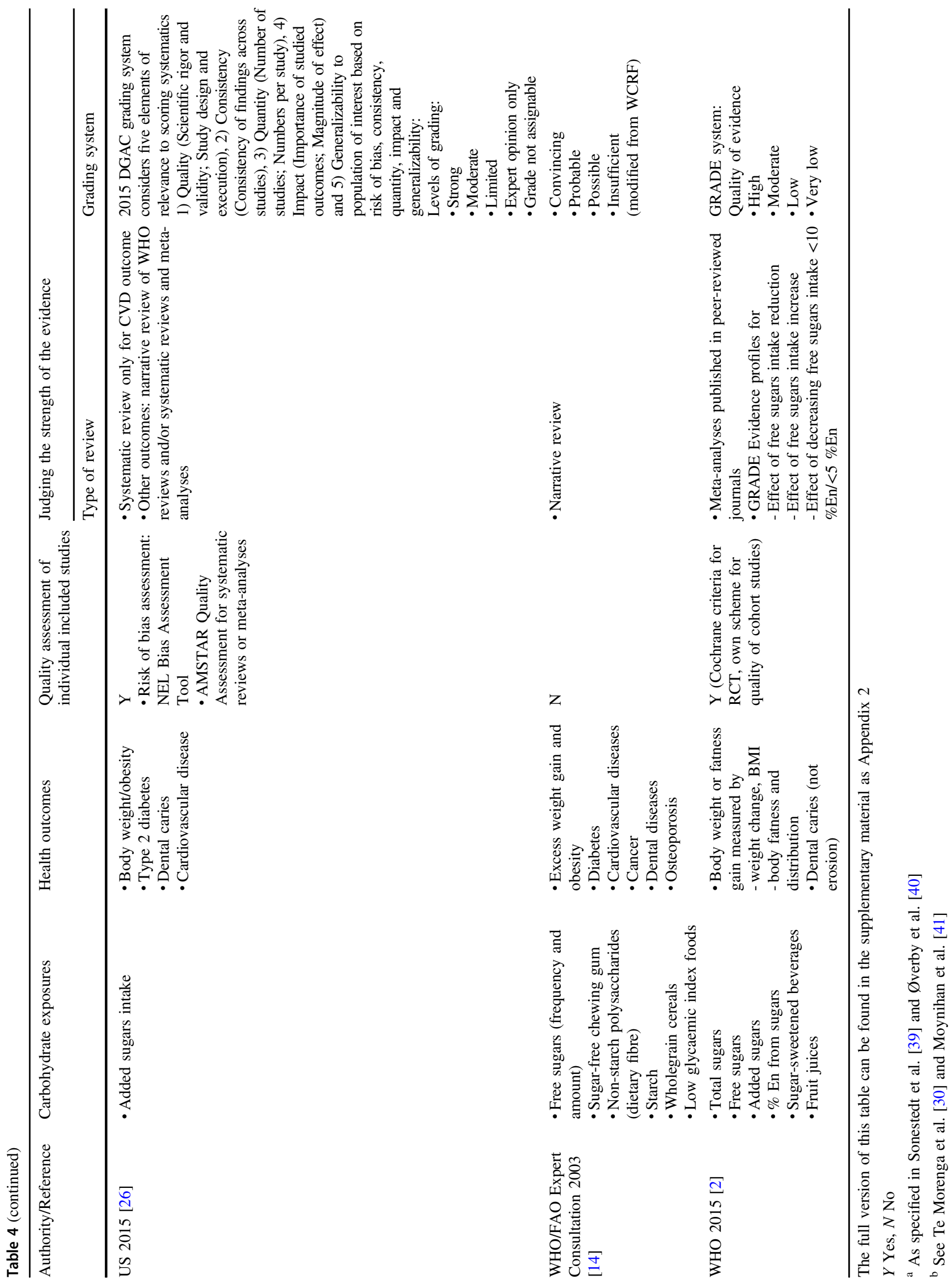


equivalent based on average energy intakes (Table 3). As with the other recommendations, the terms used for the NRs differ, including "adequate intake" [12, 22], "target value" [11], "recommended intake" [8, 14], "reference value"[9] and "nutritional goal" [17].

Although all NRs/FBGs consistently link increased fibre intake to reduced disease risks, there are significant differences in the emphasis given to particular putative health benefits. For example, most authorities assert that an adequate intake of fibre is important for normal bowel function, and cite evidence for protective effects against metabolic disease, but they differ with regard to benefits for reducing cancer risks. Thus the 2010 US report [10] cites a "moderate" body of evidence for protective effects of dietary fibre from "whole foods" against cardiovascular disease (CVD), obesity and type 2 diabetes but makes no reference to cancer. In contrast, both the UK SACN report [9] and Nordic Nutrition Recommendations (NNR) [8] give high prominence to protective effects of fibre against colorectal cancer. The UK report contained a new meta-analysis of epidemiological studies and a quantitative estimate of a dose-related protective effects of fibre against colorectal cancer, which together with similar evidence relating to CVD and type 2 diabetes, was used to derive the fibre NRs. The reports from different authorities consistently recommend that dietary fibre should come from a variety of food sources.

Table 5 Definitions of "added sugars" and their use in reports:

- Generally "sugars" are defined as "mono- and disaccharides". Accordingly, "added sugars" is often considered to be "added monoand disaccharides".

-WHO report [2]: introduced the term "free sugars" as:"all monosaccharides and di-saccharides added to foods by the manufacturer, cook, or consumer, plus sugars naturally present in honey, syrups, and fruit juices".

-US: United States Food and Drug Administration [38] and United States Department of Agriculture [16]: Added Sugars are all sugars that are either added during the processing of foods, or are packaged as such, and include sugars (free, mono- and disaccharides), syrups, naturally occurring sugars that are isolated from a whole food and concentrated so that sugar is the primary component (e.g., fruit juice concentrates), and other caloric sweeteners.

-UK: SACN report [9] adopted the term "free sugars from WHO", which now replaces the terms "added sugars" and "non- milk extrinsic sugars" (NMES), used previously. "Free sugars' comprises all monosaccharides* and disaccharides* added to foods by the manufacturer, cook or consumer, plus sugars naturally present in honey, syrups and unsweetened fruit juices. Under this definition lactose (the sugar in milk) when naturally present in milk and milk products and the sugars contained within the cellular structure of foods (particularly fruits and vegetables) are excluded".

-EU: EFSA report [12]: added sugars are "mono-, disaccharides and starch hydrolysates (e.g., glucose syrup, fructose syrup, maltodextrins) added during food preparation and manufacturing".
Glycaemic index and glycaemic load

Most authorities make no specific recommendations with regard to GI/GL $[4,5,8,9,11,12,14-16,25]$. In a few cases, even while no NRs/FBGs were made, reports include evaluations indicating relationships with specific health outcomes that would favour lower GI or GL diets [4, 7, 9, $14,26]$. Nevertheless, the absence of any explicit guidance on GI/GL in most NRs/FBGs can be attributed to the evidence being rated as inconclusive or insufficient. Other authorities judge the evidence for links of GI/GL with diseases such as type 2 diabetes as 'possible' only [7, 14], which would not justify an explicit recommendation in guidelines. The most recent FBGs from The Netherlands notes that it was unclear whether GI/GL adds value to other data on the effects of $\mathrm{CHO}$ and fibre on chronic diseases [5]. In contrast to other authorities, the Spanish and Australia/ New Zealand total carbohydrate NRs explicitly recommend that the majority of $\mathrm{CHO}$ is derived from low glycaemic index sources $[22,24]$ but with no detailed scientific justification for this.

\section{Whole grain}

There is currently no generally accepted definition of "whole grain" and "whole grain products" that can be used to provide quantitative recommendations for consumers. This problem is generally recognized [27] and attempts are being made to establish such a definition [28]. As a result, dietary guidelines for whole grain consumption remain purely qualitative (e.g., "Enjoy grain (cereal) foods, mostly whole grain and/or high cereal fibre varieties" [4], "increasing complex carbohydrates, particularly those from whole-grain cereals" [24], "diversification of the diet to provide increasing amounts of whole grains, pulses, fruits and vegetables is encouraged."[9]

\section{Methodological approaches}

We identified ten reports from eight authorities providing detailed descriptions of the evidence-based approaches used for the development of NRs/FBGs (Table 4).

\section{Exposures/outcomes}

The exposures considered in the different reports varied (Table 4, 1st column). With regard to sugars, the WHO [2] included a wide range of measures of exposure to sugars (total, added, and free) in general, but only considered fruit juices and (other) SSBs as specific sugar-providing foods. Two authorities focused primarily on sugar-containing foods [4, 18], while most other authorities followed a nutrient-based approach with additional consideration of 
some specific foods contributing most to sugar intake, such as SSB [2, 7, 9, 10, 26]. For fibre intakes, different reports gave attention to exposures from specific food sources, particularly fruits, vegetables, and (whole) grains [7, 10, 14, 26].

Regarding health outcomes, the WHO sugars report [2] considers only dental caries, body weight and fat gain. The other reports generally consider a broader range of nutrition-related diseases (Table 4, 2nd column; "health outcomes").

\section{Identification and aggregation of source materials}

Earlier reports such as those from EFSA in 2010 [12] and WHO/FAO in 2003 [14] do not provide information on the time period of evidence considered, the criteria for included/ excluded studies, or the search strategies used. A systematic quality assessment of the individual studies included in these reports was not performed and the overall strength of the evidence was judged in a purely narrative way.

More recent reports have predominantly been based on a comprehensive (systematic) literature search, with most authorities using multiple databases (see Appendix 2, "search strategy") to identify relevant original studies or systematic reviews published within a stated timeframe prior to the report publication (see Appendix 2, "time period"). These reports generally consider evidence only from intervention trials and prospective cohort studies [2, 7-10, 26]. (see Appendix 2, "studies included/excluded").

The newer reports are consistently based on systematic reviews, and the WHO sugars report [2] and UK SACN Report [9] also commissioned their own meta-analyses.

\section{Quality and grading assessments applied}

The type of quality assessment of the individual studies included in the reports differed across authorities, ranging from a limited quality check, or use of the WHO or European Association for the Study of Obesity schemes [29] to the use of own schemes [2, 4, 8, 10, 26] (Table 4, 3rd column). The US scheme [10, 26] in particular is very comprehensive, addressing the extent to which study design and conduct were shown to be protected from systematic bias, non-systematic bias, and inferential error. The related classifications for these were "strong", "moderate" and "limited" evidence (or "not assignable"). Qualitative descriptions developed for each of these elements leave no space for interpretation differences. Such detailed information on quality assessment is absent in other cases.

The totality of evidence for specific diet-health relationships was generally graded in accordance with the WHO scheme (convincing/probable/possible/insufficient)
[2, 4, 7, 8, 14] or similar differently worded but similar schemes [9, 10, 26], (Table 4, 4th column).

\section{Public consultation}

Most authorities presented a draft version of their report for input from the general public including professional and trade organizations, before preparing a final version (see Appendix 2, "public consultation").

\section{Implementation}

The authorities from Australia, Germany, the Netherlands and the Nordic countries [4, 7, 8, 18] specified that NRs/ FBGs for the general public should be restricted to nutrientdisease relationships with a strong evidence base only (according to their grading systems) (see Appendix 2, "specific considerations for implementation"). The Australian NHMRC report states that implementation should also consider other sources of evidence [4]. The Nordic report (NNR) requests that advice on implementation fits into the whole-diet approach and includes consideration of current dietary practices [8]. The final documents giving advice directly to the public are often produced by groups other than those undertaking the evidence assessment, and commonly draw on additional sources of evidence and/or considerations [4, 5, 7-9, 16]. The WHO recommendations for implementation build on a broad set of additional specific public health nutrition considerations that can vary by region [2]. In the $\mathrm{UK}$ the implementation of the expert recommendations from SACN [9] is primarily the responsibility of Public Health England. In the Netherlands, the FBGs were developed by the Health Council [5], whereas the Dutch Nutrition Centre (Voedingscentrum) is the authority that implements these into practical guidelines for the public.

\section{Discussion}

In the countries and regions considered here, recent dietary NRs/FBGs with respect to total $\mathrm{CHO}$ and its components have many general similarities, particularly in recommending a relatively high total $\mathrm{CHO}$ and fibre intake and limited intake of (added or free) sugars. Nonetheless, some notable differences exist in the NRs/FBGs, and particularly in the methods used to derive them. It is relevant to ask why such differences arise, given a common global evidence base.

There are several important differences between the present analysis and a recent review by Erickson et al [6]. That work considered only sugars recommendations, and not total carbohydrate, fibre or other aspects of dietary 
carbohydrates, and also differed in the specific regions included. Most importantly, Erickson et al. [6] focused on assessing and grading the methodological quality of guidelines and supporting evidence. In contrast, we focused on reporting the overall approaches followed by different authorities, in order to consider the extent to which the differences in these approaches were reflected in variation amongst their resulting recommendations. Whereas Erickson et al. [6] conclude that the quality of available evidence linking sugars with health outcomes was low, we note relatively high consistency in international NRs/FBGs for carbohydrate quality and quantity, despite the differing approaches applied.

Significant differences in published NRs and FBGs would be problematic for several reasons. Through traditional and social media, consumers will now quickly hear if "health messages" appear to be different or conflicting. This can encourage the view that there is poor consensus or consistency in the expert community, and adversely impact the acceptance and implementation of recommendations. Differences in NR and FBGs can also have implications for the food and beverage industry, creating a challenge to implementation of consistent actions and messages from multinationals and trade organizations that operate across multiple regions. In the sections below, specific elements of the processes applied in generating NRs and FBGs are discussed.

\section{Which exposures and health outcomes were considered?}

With respect to exposures, differences in the definition of sugar intake are most notable (Table 5). This reflects growing recognition that it is not just the sugars themselves but the source or matrix in which they appear that should be considered in interpreting exposure data, prompting an emphasis on "free" or "added" vs. just "total sugars". For derivation of NRs, the a priori specification of differing specific exposures will in turn affect the subsequent selection of publications for the evidence appraisal. Hence for consistency there is a need to base the evidence appraisal on common exposure definitions, clearly for sugars but also for whole grains, and perhaps also for fibres not naturally occurring intact in foods (e.g., isolated, extracted, and modified fibres).

A recent shift in the focus of interest from NRs to FBGs and/or recommendations for a healthy dietary pattern is also reflected in the development of the reports. Most reports now include explicit assessments of evidence linking foodspecific exposures to health outcomes. With respect to $\mathrm{CHO}$ nutrition, this review illustrates a majority of authorities considered only whole grain and/or sources of dietary sugars (mostly SSB) as food-based exposures [2, 7, 14].
However, the Australian and most recent Dutch guidelines focus exclusively on food-based exposures $[4,5]$. The 2015 US report takes the food-based approach one step further in focusing on dietary patterns in relation to health outcomes, postulating that the totality of diet, i.e. the combinations and quantities in which foods and nutrients are consumed, may have synergistic and cumulative effects on health and disease [26]. This approach, however, limits making conclusions on the relevance of specific nutrients or (CHO-rich) foods.

A main challenge for future reports will likely lie in the development of conceptual approaches that utilize and combine the wealth of physiological evidence available for nutrient-disease links together with emerging data on the public health relevance of consuming specific foods or dietary patterns. As an example, evidence linking SSBs (as a food-based exposure) to health outcomes can be clearly separated from evidence linking dietary sugar (as a nutrientbased exposure) to the same outcomes. This is reflected for example in the German and UK reports, which link SSBs but not total sugars or sucrose to risk of diabetes [7, 9].

To confidently draw out health relationships with specific foods requires that intakes of those foods are sufficiently and reliably defined, quantifiable, variable and widespread, such that associations can be robustly tested and (specific) FBGs founded on these. Few foods meet all these criteria but SSBs are a good example; furthermore, as most SSBs are also $100 \%$ En from (added/free) sugars, there is a close alignment with sugar NRs. In contrast, many other major sources of added/free sugars in the diet (e.g., cakes, cookies, confectionery) derive a high proportion of energy from starches and fats. As a result many FBGs are not based on direct evidence of health relationships for each specific food, but reflect exposure data aggregated for a class of foods, or the general application of nutrient-specific data to important individual food sources of that nutrient.

The guidance documents reviewed here invariably state that the NRs are for application in the general population, and may not apply to groups or individuals with specific diseases or differing nutritional needs. The most prevalent diet-related diseases are considered across many of the reports, but these each differ in which specific health outcomes have been selected for evidence appraisal and, especially, in the outcomes used as the basis for quantitative NRs. Resource constraints, public health priorities, or hypotheses related to particular outcomes may often be reasons for focusing on a particular outcome set. Body weight and obesity are prominent health issues that have been considered in almost all reports, and in relation to all of the carbohydrate exposures considered here. Diabetes is similarly prevalent in the reports as an outcome of interest for all carbohydrate exposures. Other outcomes are more exposure-specific; e.g., colo-rectal cancer for fibre, or 
overall dietary patterns or quality for sugars. Nevertheless, even where similar outcomes were assessed, those used as the basis for recommendations often differed. This is most apparent in regard to the quantitative NRs on sugars from WHO [2], US (DGAC) [10] and UK (SACN) [9], where similar NRs were derived from quite different parts of the overall evidence. Likewise, the justifications for the (relatively similar) numerical ranges/values for NRs on total CHO vary (Table 1$)$.

\section{How were data selected?}

Variations in the process of systematic search and selection of evidence generally appeared to have a rather limited effect on the NR/FBG. All reports applying a systematic approach searched similar databases up to a contemporary cut-off date, and considered mainly cohort and intervention studies. Nevertheless, specific inclusion criteria did vary somewhat, leading to potentially important differences in the evidence bases available for different assessments. For example, when selecting evidence testing the relationship of sugar intakes with body weight change, the WHO applied a criterion of a minimum 2 weeks and 1-year duration respectively for intervention trials and prospective cohort studies [30], whereas the UK report [9] had corresponding criteria of 1 and 3 years.

The nature of quality assessment of the individual studies included in the reports also differed, with nutrition evidence library (NEL) [31, 32] system appearing to be the most comprehensive. Whilst it would be desirable for other authorities to perform similar in-depth assessments, this may not always be feasible in terms of the time, personnel and budget needed. Instead, ways of incorporating the assessment performed in the US into other national reports and/or means of co-operation may be desirable alternatives.

\section{How were data aggregated?}

This review illustrates that substantial progress has been made since the early 2000s towards more systematic approaches for evidence appraisal: While early reports consisted of purely narrative reviews (e.g., reports from WHO and EFSA) $[12,14]$ recent reports undertook or relied on published systematic reviews and meta-analyses. Nonetheless, new meta-analyses were only performed by a few authorities, to answer specific questions. Generally, the use of very recent or new meta-analyses is preferable, particularly in fields with newly emerging evidence, although considerable differences in the quality of metaanalyses have been noted [33], which needs further attention.

\section{How was the overall evidence graded?}

The grading systems for judging the overall evidence for specific diet-disease associations also differed amongst panels that performed the assessments. This may reflect a view that the available schemes initially developed for evidenced-based medicine (e.g., GRADE system) have some shortcomings for application in nutrition. In particular, an emphasis on RCTs with disease endpoints is unrealistic in nutrition, where most RCTs have intermediate markers as primary outcomes, and relationships with disease endpoints are predominantly derived from observational studies. Furthermore, true blinding, "dose-response" data and designs limiting treatment differences to a single component are often not feasible for RCTs of foods or diets. Given these limitations, some authorities developed their own schemes $[9,10,26]$. Other authorities used modified versions of the World Cancer Research Fund International (WCRF) scheme [7, 8, 14]. Generally, the schemes that were applied considered both the type of evidence available (i.e., the study design and/or the risk of bias) and the consistency and quantity of the available evidence. Nonetheless, concern has been raised that a more critical appraisal of methodological issues such as confounding or collinearity (e.g., the extent to which high fibre consumption reflects a generally healthy lifestyle) for observational studies or limited consideration of effectiveness in intervention studies need to be incorporated into current schemes and/or their presentation.

An additional issue is the risk that those involved in the preparation of reports may not fully or consistently adhere to their own grading system. For example, in a qualitative analysis of interviews, Alexander et al [34]. examined why WHO report developers draw conclusions stronger than would be consistent with the GRADE scheme. Reasons included scepticism about the value of making conditional (weaker) recommendations, political considerations, high certainty in benefits (warranted or not) despite assessing evidence as low certainty, and concerns that conditional recommendations will be ignored [34]. Similar concerns may apply to the adherence of other expert bodies using other grading systems. Hence, maximizing adherence to grading systems through involvement of experts in their use, and training and monitoring of content experts' use of the grading systems, may reduce a source of "noise" in the interpretation of evidence. The extent to which such measures were implemented could not readily be extracted from the reports considered in the present review. Thus, it seems relevant to recommend greater transparency in the roles of methodologists and content experts, and emphasis on measures to separate the processes applied in the report development. 


\section{How was evidence translated into (regional) guidelines?}

We noted that in several regions and the WHO $[2,4,5,7-9$, 16], the responsibility for the implementation and/or translation into national NRs/FBGs as policies intended for the public ("risk management") sits with authorities not directly involved in the assessment and reporting of the evidence ("risk assessment"). Nonetheless, some terminology used by the experts in the assessment of the evidence does give general guidance as to which level of evidence should be considered when deriving the NRs/FBGs for implementation (e.g., Evidence grades A-C in Australia [4], evidence grades "convincing" or "probable" in German (DGE) or Nordic Nutrition Recommendations) [7, 8].

Even where the evidence base and grading systems are similar, the local context could give rise to differing NRs/ FBGs, reflecting for example an appreciation of the effect sizes of the observed associations against the local prevalence/incidence rates of the diseases as well as the local intake levels (e.g., of dietary sugars and fibre). In reality, such differentiation based on the local conditions generally was not apparent. In terms of $\mathrm{CHO}$ nutrition, intake levels do not vary greatly between the populations in the countries considered in this review, nor do the major diet-related diseases of concern [8, 9, 24, 25, 35]. Furthermore, for example, the US analyses of three different 'healthy' diet patterns all produced similar quantitative limits for added sugars that would be compatible with meeting the other food group and nutrient recommendations [13]. Given this convergence of information, it is perhaps not surprising that justifications for (local) NRs/FBGs on sugars and fibre intake do not specifically refer to local population intake levels, but are instead driven by more general considerations regarding (i) diet-disease associations and (ii) nutrient adequacy (e.g., restriction of added sugar intakes to ensure adequate intakes of micronutrients and dietary fibre).

A final point to consider is whether the quantitative NRs are set to be applied at the individual or population level. In some cases the NRs are clearly conveyed as population means or targets $[2,9,14]$. In other cases there is a degree of ambiguity about this (e.g., NNR [8], US (DGAC) report [13], Australia/New Zealand NHMRC report [22]). In principle, this could be an important issue and therefore deserves more clarity. For example, for added or free sugars, a recommended upper value intended for individuals implies a much more conservative mean population value. In practice, however, population recommendations tend to be understood and communicated as goals for individuals, even if this was not the stated intent.

\section{General conclusions}

Overall, despite many similarities in recommending a relatively high total $\mathrm{CHO}$ and fibre intake and limited intakes of (added or free) sugars, the justifications for CHOrelated NRs/FBGs differ across a range of national and international authorities. We identified a number of differences in the underlying definitions of $\mathrm{CHO}$ exposures, the relative emphasis on nutrients vs. foods, and the choice of health outcomes as dominant sources of differences in the evidence appraisal. However, differences in the selected research papers, time frames and method used for data aggregation (review/meta-analysis) appear to have been (perhaps surprisingly) a rather minor source of variation in $\mathrm{NRs/FBGs.} \mathrm{It} \mathrm{is} \mathrm{possible} \mathrm{that} \mathrm{variation} \mathrm{in} \mathrm{grading} \mathrm{systems}$ and adherence to these, as well as an incomplete separation of methodological from content-related processes, may also have contributed to some of the differences; however information on these is sparse in the respective reports and a more transparent presentation of those processes is needed. We acknowledge this review was limited by geography and language, though it seems likely our conclusions would also be relevant to the work of authorities not included here.

Taken together, this review underlines the likely value of more explicit quantitative justifications for numerical recommendations and/or the explicit communication of uncertainty (conditional recommendations). This will enhance the credibility and adoption of NRs, and their translation into widely accepted, well-coordinated (global) public health initiatives. To minimize differences in the evidence appraisal, harmonization is particularly warranted with regard to the underlying definitions of $\mathrm{CHO}$ exposures, as well as the consideration of a wide range of relevant nutrition-related outcomes.

Acknowledgements This work was conducted by an expert group convened by the European branch of the International Life Sciences Institute, ILSI Europe. This expert group carried out the work, i.e., collecting/analysing data/information and writing the scientific paper. The related meetings were coordinated by the ILSI Europe Dietary Carbohydrates Task Force. The authors' responsibilities were as follows: JS and FB conceived the project. Data search and first screen of all 1781 retrieved papers was performed by DJM with IJ. Review of the selected papers for fitting defined criteria was performed by IJ, AEB and FB. Methodologies were extracted by AEB. Extraction of the recommendation justifications, when given, was done by IAM, FB and DJM. Specific recommendations on wholegrain, dietary GI and dietary GL were extracted by DJM, IJ and FB. AEB and FB wrote the first draft of the manuscript. All authors contributed to the interpretation of the results, critically revised the manuscript, and approved the final version of the paper. We gratefully acknowledge the contribution of Dr A.Piekarz (SA Coca-Cola Services NV, Brussels, Belgium) and Dr Stephan Theis (Südzucker Group, Obrigheim, Germany) in selection of relevant documentation and critical inputs.

Conflict of interest This work was conducted by an expert group of the European branch of the International Life Sciences Institute, ILSI 
Europe. This publication was coordinated by the Dietary Carbohydrates Task Force. Authors DJM and JDS are employed by commercial organizations that manufacture carbohydrate-containing foods and beverages or ingredients used in making these. The expert group received funding from the ILSI Europe the Dietary Carbohydrates Task Force members. Industry members of this task force are listed on the ILSI Europe website at http://ilsi.eu/task-forces/nutrition/dietary-ca rbohydrates/. Experts are not paid for the time spent on this work; however, the non-industry members within the expert group were offered support for travel and accommodation costs from the Dietary Carbohydrates Task Force to attend a meeting to discuss the manuscript and a small compensatory sum (honoraria) with the option to decline. The research reported is the result of a scientific evaluation in line with ILSI Europe's framework to provide a precompetitive setting for public-private partnership (PPP). ILSI Europe facilitated scientific meetings and coordinated the overall project management and administrative tasks relating to the completion of this work. The opinions expressed herein and the conclusions of this publication are those of the authors and do not necessarily represent the views of ILSI Europe nor those of its member companies.

Open Access This article is licensed under a Creative Commons Attribution-NonCommercial-NoDerivatives 4.0 International License, which permits any non-commercial use, sharing, distribution and reproduction in any medium or format, as long as you give appropriate credit to the original author(s) and the source, and provide a link to the Creative Commons license. You do not have permission under this license to share adapted material derived from this article or parts of it. The images or other third party material in this article are included in the article's Creative Commons license, unless indicated otherwise in a credit line to the material. If material is not included in the article's Creative Commons license and your intended use is not permitted by statutory regulation or exceeds the permitted use, you will need to obtain permission directly from the copyright holder. To view a copy of this license, visit https://creativecommons.org/licenses/by-nc-nd/4. $0 /$

\section{References}

1. World Health Organization. Global status report on noncommunicable diseases. Geneva, WHO; 2014.

2. World Health Organization. Guideline: sugars intake for adults and children. Geneva, WHO; 2015.

3. World Health Organization. Global action plan for the prevention and control of noncommunicable diseases 2013-2020. WHO, Geneva; 2013.

4. National Health and Medical Research Council. Australian dietary guidelines. National Health and Medical Research Council, Canberra; 2013

5. Health Council of the Netherlands. Dutch dietary guidelines 2015 publication no. 2015/24E. Health Council of the Netherlands, The Hague; 2015

6. Erickson J, Sadeghirad B, Lytvyn L, Slavin J, Johnston BC. The scientific basis of guideline recommendations on sugar intake: a systematic review.Ann Intern Med 2017;166:257-267.

7. Hauner H, Bechthold A, Boeing H, Bronstrup A, Buyken A, Leschik-Bonnet E, et al. Evidence-based guideline of the German nutrition society: carbohydrate intake and prevention of nutritionrelated diseases. Ann Nutr Metab. 2012;60(Suppl 1):1-58.

8. Nordic Council of Ministers. Nordic nutrition recommendations (NNR) 2012: integrating nutrition and physical activity. Copenhagen, Norden; 2012.

9. Scientific Advisory Committee on Health (SACN). Carbohydrates and Health. London, The Stationery Office; 2015.
10. US Department of Agriculture and US Department of Health and Human Services. Report of the Dietary Guidelines Advisory Committee on the Dietary Guidelines for Americans 2010. Washington D.C., US Government Printing Office; 2010.

11. Deutsche Gesellschaft für Ernährung, Österreichische Gesellschaft für Ernährung, Schweizerische Gesellschaft für Ernährungsforschung, Schweizerische Vereinigung für Ernährung (D-A-CH) Referenzwerte für die Nährstoffzufuhr. Deutsche Gesellschaft für Ernährung (DGE), Österreichische Gesellschaft für Ernährung (ÖGE), Schweizerische Gesellschaft für Ernährung (SGE): Bonn. https://www.dge.de/wissenschaft/referenzwerte/. 2012.

12. EFSA Panel on Dietetic Products, Nutrition and Allergies (NDA). Scientific opinion on dietary reference values for carbohydrates and dietary fibre. EFSA J 2010;8:1462.

13. US Department of Agriculture, US Department of Health and Human Service, Dietary Guidelines Advisory Committee. Scientific Report of the 2015 Dietary Guidelines Advisory Committee. Washington D.C., US Government Printing Office; 2015.

14. World Health Organization. Diet, nutrition and the prevention of chronic diseases. Report of a joint WHO/FAO expert consultation. Geneva,WHO; 2003.

15. Health Council of the Netherlands. Dietary reference intakes: energy, proteins, fats and digestible carbohydrates. The Hague, Health Council of the Netherlands; 2001.

16. US Department of Health, Human Services, US Department of Agriculture. 2015-2020 Dietary guidelines for Americans. Washington D.C., US Government Printing Office; 2015.

17. Institute of Medicine (IoM). Dietary reference intakes for energy, carbohydrate, fiber, fat, fatty acids, cholesterol, protein, and amino acids (macronutrients). Washington, DC, The National Academies Press; 2005.

18. Health Council of the Netherlands. Background document methodology for the evaluation of the evidence for the Dutch dietary guidelines 2015. Publisher. The Hague, Health Council of the Netherland; 2015.

19. Gezondheidsraad. Verteerbare koolhydraten. Achtergronddocument bij Richtlijnen goede voeding 2015. Den Haag; 2015.

20. Gezondheidsraad. Voedingsvezel. Achtergronddocument bij Richtlijnen goede voeding 2015. Den Haag; 2015.

21. Health Council of the Netherlands. Guideline for dietary fibre intake. The Hague: Health Council of the Netherlands, 2006; publication no. 2006/03E. ISBN 90-5549-604-9.

22. National Health and Medical Research Council (NHMRC), Australian Government Department of Health and Ageing, New Zealand Ministry of Health. Nutrient reference values for Australia and New Zealand. Canberra, National Health and Medical Research Council; 2006.

23. Serra-Majem L, Aranceta J. Nutritional objectives for the Spanish population. Consensus from the Spanish Society of Community Nutrition. Pub Health Nutr 2001;4:1409-1413.

24. Aranceta J, Serra-Majem L. Dietary guidelines for the Spanish population. Pub Health Nutr 2001;4:1403.

25. Food Safety Authority of Ireland (FSAI). Scientific recommendations for healthy eating guidelines in Ireland. Dublin; 2011. ISBN 19-0446-576-5, ISBN 97-8190-446-576-8.

26. US Department of Health and Human Services, US Department of Agriculture, Dietary Guidelines Advisory Committee. Scientific Report of the 2015 Dietary Guidelines Advisory Committee. Part C: Methodology. Washington, D.C., US Government Printing Office; 2015.

27. Seal CJ, Nugent AP, Tee E-S, Thielecke F. Whole-grain dietary recommendations: the need for a unified global approach. Br J Nutr 2016;115:2031-2038.

28. Van Der Kamp JW, Poutanen K, Seal CJ, Richardson DP. The healthgrain definition of "whole grain". Food Nutr Res 2014;58:22100. 
29. Tsigos C, Hainer V, Basdevant A, Finer N, Fried M, MathusVliegen E, et al. Management of obesity in adults: European clinical practice guidelines. Obes Facts 2008;1:106-116.

30. Te Morenga L, Mallard S, Mann J. Dietary sugars and body weight: systematic review and meta-analyses of randomised controlled trials and cohort studies. BMJ 2012;346:e7492.

31. US Department of Health and Human Services, US Department of Agriculture Dietary Guidelines Advisory Committee. Carbohydrates. http://www.nel.gov/topic.cfm?cat2854. 2010.

32. US Department of Health and Human Services and US Department of Agriculture Dietary Guidelines Advisory Committee. Added sugars risk of cardiovascular disease. Washington, D.C., US Government Printing Office; 2015.

33. Greco T, Zangrillo A, Biondi-Zoccai G, Landoni G. Meta-analysis: pitfalls and hints. Heart Lung Vessel 2013;5:219-225.

34. Alexander PE, Gionfriddo MR, Li S-A, Bero L, Stoltzfus RJ, Neumann I, et al. A number of factors explain why WHO guideline developers make strong recommendations inconsistent with GRADE guidance. J Clin Epidemiol 2016;70:111-22.

35. Centers for Disease Control and Prevention. National Health and Nutrition Examination Survey. http://www.cdc.gov/nchs/nhanes/. 2017.
36. Food and Nutrition Board: Institute of Medicine. Dietary reference intakes for energy, carbohydrate, fiber, fat, fatty acids, cholesterol, protein and amino acids (Macronutrients). Washington, DC: National Academy Press; 2002.

37. Keller U, Battaglia-Richi E, Beer M, Darioli R, Meyer K, Renggli A et al. Schweizerischer Ernährungsbericht. Bern, Bundesamt für Gesundheit, 2012.

38. US Food and Drug Administration. Changes to the nutrition facts label. 2016. http://www.fda.gov/Food/GuidanceRegulation/Guida nceDocumentsRegulatoryInformation/LabelingNutrition/ ucm385663.htm.

39. Sonestedt E, Overby NC, Laaksonen DE, Birgisdottir BE. Does high sugar consumption exacerbate cardiometabolic risk factors and increase the risk of type 2 diabetes and cardiovascular disease? Food Nutr Res 2012;56:19104.

40. Øverby NC, Sonestedt E, Laaksonen DE, Birgisdottir BE. Dietary fiber and the glycemic index: a background paper for the nordic nutrition recommendations 2012. Food Nutr Res 2013;57:20709.

41. Moynihan PJ, Kelly SAM. Effect on caries of restricting sugars intake: systematic review to inform WHO guidelines. J Dent Res 2014;93:8-18. 